\title{
Fuerza de asociación entre manifestaciones depresivas y estado nutricional en adultos mayores institucionalizados
}

\section{Strength of association between depressive manifestations and nutritional} status in institutionalized older adults

\author{
Josselyne Escobedo-Encarnación ${ }^{1}$ \\ Elsa Zavala-Palacios ${ }^{1}$ \\ María del Carmen Taipe-Aylas ${ }^{2}$ \\ Frank Peralta-Alvarez ${ }^{3}$
}

\begin{abstract}
Resumen
Objetivo: determinar la fuerza de asociación entre manifestaciones depresivas y estado nutricional en adultos mayores institucionalizados de Lima y Callao. Materiales y métodos: se realizó un estudio de alcance explicativo y de corte transversal. La muestra fue conformada por 128 individuos mayores de 60 años, de los cuales el 50.8\% fueron mujeres y 49.2\% hombres. Las manifestaciones depresivas se evaluaron mediante la Escala de Depresión Geriátrica Yesavage, versión reducida (GDS, por las siglas en inglés) y el estado nutricional se determinó a través de la Evaluación del Mini Nutrition Assesment. Se realizó un análisis inferencial para establecer la asociación y fuerza de asociación de las variables. Asimismo, los datos fueron analizados mediante el Chi Cuadrado y el modelo de Regresión Logística con el programa estadístico Stata versión 13. Resultados: se encontró asociación entre ambas variables ( $<<0.001$ ); y las manifestaciones depresivas se asociaron positivamente con la presencia de ries go de malnutrición OR 7.91 (IC 95\% 3.1319.9). Los resultados obtenidos no son extrapolables a la población adulta mayor en comunidades; sin embargo, son válidos para poblaciones de adultos mayores institucionalizados. Conclusión: las manifestaciones depresivas y el sexo femenino están fuertemente asociados con el riesgo de malnutrición en los adultos mayores institucionalizados .

Palabras clave: Depresión; Estado nutricional; Adulto mayor (Fuente: DeCS).
\end{abstract}

\begin{abstract}
Objective: to determine the strength of association between depressive manifestations and nutritional status in institutionalized older adults in Lima and Callao. Materials and methods: an explanatory and cross-sectional study was conducted. The sample was made up of 128 individuals over 60 years, of which $50.8 \%$ were women and $49.2 \%$ men. Depressive manifestations were evaluated using the Yesavage Geriatric Depression Scale, reduced version (GDS) and nutritional status was determined through the Mini Nutrition Assessment. An inferential analysis was performed to establish the association and strength of association of the variables. Likewise, the data were analyzed using the Chi Square and the Logistic Regression model with the statistical program Stata version 13. Results: an association was found between both variables ( $\mathrm{p}<0.001$ ); and depressive manifestations were positively associated with the presence of risk of malnutrition OR 7.91 (95\% CI 3.13-19.9). The results obtained are not extrapolated to the elderly population in communities; however, they are valid for institutionalized elderly populations. Conclusion: depressive manifestations and female sex are strongly associated with the risk of malnutrition in institutionalized older adults .
\end{abstract}

Keys words: Depression; Nutritional status; Elderly (Source: DeCS).

Para citar:

Escobedo E, Zavala E, Taipe M, Peralta F. Fuerza de asociación entre manifestaciones depresivas y estado nutricional en adultos mayores institucionalizados. CASUS. 2019;4(3):170-175.

DOI: 10.35626 /casus.3.2019.220

\footnotetext{
${ }^{1}$ DIRIS Lima Norte MINSA. Lima, Perú.

${ }^{2}$ Universidad Científica del Sur. Lima, Perú.

${ }^{3}$ Universidad Peruana Cayetano Heredia. Lima, Perú.

Correo electrónico: jos selyneescobedo@gmail.com
}

Fecha de recepción: 05-06-17

Fecha de envío a pares: 10-06-17

Fecha de aprobación por pares: 15-11-19

Fecha de aceptación: 06-12-19 


\section{INTRODUCCIÓN}

Según la Organización Mundial de la Salud (OMS) la población senil aumentará entre el periodo 2000 al 2050 (1), envejeciendo de manera acelerada en los próximos años (1). Asimismo, la cantidad de adultos mayores se duplicará de un $11 \%$ a un $22 \%$ en los siguientes 15 años (2,3). Esta etapa de la vida es vulnerable y propensa a diferentes cambios en su estado nutricional, tanto en países de ingresos económicos bajos como en los altos (4). Frente a ello, se considera la importancia de la temática del envejecimiento y la identificación de los factores que ayuden a su prevención.

La etapa del envejecimiento es considerada como un proceso de continuos cambios que repercuten en el estado de salud. En esta etapa de vida del adulto mayor se acrecienta el riesgo de presentar deficiencias nutricionales a causa de diversos factores. En el Perú, el grupo de los adultos mayores constituye el $9.1 \%$ de la población (5). Mientras que, la desnutrición es uno de los problemas nutricionales percibidos en esta población que pudiera afectar su estado de salud. De hecho, la prevalencia de desnutrición se encuentra entre el 1 al $15 \%$ en adultos mayores ambulatorios, del $17 \%$ al $70 \%$ en los hospitalizados y es del $85 \%$ en los que residen en asilos (6).

Al respecto, existen diversos factores de riesgo para desarrollar malnutrición en la población adulta mayor que a su vez pueden ser modificados por medio de la intervención (7). Entre ellos se encuentran la situación familiar, la dependencia funcional, las enfermedades crónicas, la demencia y la depresión. Entre los mencionados la presencia de manifestaciones depresivas resulta relevante debido a su prevalencia en esta etapa de vida. Se estima que aproximadamente una de cada cinco personas pudiera desarrollar un cuadro depresivo a lo largo de su vida (8).

A nivel mundial, la incidencia de depresión es hasta dos veces más alta en mujeres que en hombres (9). Este diagnóstico en personas adultas mayores se manifiesta conectado a la ausencia de recuperación de enfermedades, a una mayor estancia hospitalaria, a la pérdida de algún familiar, al abandono social, entre otros (6,9). Es así que estos factores juegan un papel importante en la presencia de malnutrición en este grupo etario. Por estos motivos los problemas nutricionales deben tenerse en cuenta. Además, en el Perú existe un alto porcentaje de manifestaciones depresivas y malnutrición en el adulto mayor institucionalizado frente aquellos que se encuentran hospitalizados y los que residen en comunidad (6).

Sin embargo, en la actualidad son escasos los estudios nacionales que establezcan la fuerza de asociación entre ambas variables en la población adulta mayor institucionalizada. A partir de la situación descrita el estudio tuvo como objetivo determinar la fuerza de asociación entre manifestaciones depresivas y estado nutricional en adultos mayores institucionalizados de Lima y Callao.

\section{MATERIALES Y MÉTODOS}

Se realizó un estudio correlacional y explicativo de corte transversal (10). Se llevó a cabo un censo de 128 adultos mayores de ambos sexos desde los 60 años a más e institucionalizados. No se consideraron aquellos adultos mayores con deterioro cognitivo determinado a través de la Escala de Pffeifer. Esta escala se aplicó previamente para determinar el estado cognitivo, cuya sensibilidad es de $100 \%-68 \%$ y especificidad de un 90\%-96\% (11). Los datos fueron recolectados durante un período de cinco meses.

La presencia de manifestaciones depresivas fue medida a través de la Escala de Depresión Geriátrica Yesavage, versión reducida (GDS en sus siglas en inglés). Esta herramienta es usada como parte del formato de atención en evaluación del adulto mayor establecido en la Norma Técnica de Salud para la Atención Integral de Salud de las Personas Adultas Mayores NTS $\mathrm{N}^{\circ} 043-$ 2006/MINSA (12). La escala no se ha modificado hasta la actualidad a nivel nacional en las instituciones MINSA y no MINSA y continúa en uso. Además, ha sido validada en versión reducida en el consultorio externo de geriatría del Hospital 
Nacional Cayetano Heredia, con una sensibilidad de $93 \%$, y una especificidad de $78 \%$ (13). La clasificación reducida considera las siguientes categorías: $\sin$ manifestaciones depresivas (0-1 punto), con manifestaciones depresivas (3-4). Esta variable fue clasificada como dicotómica con una escala de medición ordinal.

Para determinar el estado nutricional se aplicó la Escala Mini Nutritional Assessment (MNA), la cual fue creada a principios del año 1990 y fue desarrollada y validada por los investigadores del equipo geriátrico de Toulouse en Francia (14). La misma, fue adaptada y validada en Perú con una sensibilidad de $96 \%$ y especificidad $98 \%$ (15) y es usada como parte la Guía Técnica para la valoración nutricional antropométrica de la persona adulta mayor R.M. N²40-2013/MINSA (16). Esta escala se clasifica en: estado nutricional normal (24-30 puntos), riesgo de malnutrición (1723.5 puntos) y malnutrición ( $<17$ puntos). La variable fue clasificada como categórica ordinal.

El análisis de la información se determinó mediante el paquete estadístico Stata Versión 13. En la estadística descriptiva se aplicó para las variables cuantitativas promedios y desviaciones estándar, y frecuencias y porcentajes para las cualitativas. La estadística inferencial utilizando la prueba estadística de Chi Cuadrado.

Para determinar la fuerza de asociación entre ambas variables se realizó el análisis multivariado a través del cálculo de odds ratio (OR). Se ajustó considerando algunas variables confusoras: sexo, edad y nivel educativo para comprobar si estas influyen junto con la variable independiente sobre la variable dependiente para lo cual se realizó una regresión logística. Para todas las pruebas estadísticas se consideró un nivel de significancia del $5 \%$ y un nivel de confianza del $95 \%$.

En relación a los aspectos éticos, el estudio fue realizado bajo la autorización y aprobación por parte de un Comité de Ética Institucional. Los derechos de los participantes de la investigación fueron garantizados mediante la privacidad en el manejo de la información y el consentimiento informado.

\section{RESULTADOS}

De los 128 adultos mayores, se encontraron porcentajes similares para ambos sexos y la edad promedio fue de 79.6 años. Por otro lado, la categoría riesgo de malnutrición presentó el mayor porcentaje $(65.63 \%)$ y prevaleció el grupo con manifestaciones depresivas $(53.91 \%)$. El resto de los resultados se puede consultar en la tabla 1 .

\begin{tabular}{|c|c|c|}
\hline & $\mathbf{n}$ & $\%$ \\
\hline Edad $(m+D E)$ & \multicolumn{2}{|c|}{$79.6 \pm 7.8$} \\
\hline $\operatorname{Peso}(m+D E)$ & \multicolumn{2}{|c|}{$57.3 \pm 12.7$} \\
\hline Talla $(m+D E)$ & \multicolumn{2}{|c|}{$150.6 \pm 11.5$} \\
\hline IMC & \multicolumn{2}{|c|}{$22.5 \pm 5.3$} \\
\hline \multicolumn{3}{|l|}{ Sexo } \\
\hline Masculino & 63 & 49.22 \\
\hline Femenino & 65 & 50.78 \\
\hline \multicolumn{3}{|l|}{ Nivel Educativo } \\
\hline Primaria & 43 & 33.59 \\
\hline Secundaria & 41 & 32.03 \\
\hline Superior & 31 & 24.22 \\
\hline \multicolumn{3}{|l|}{ MNA } \\
\hline Normal & 39 & 30.47 \\
\hline $\begin{array}{l}\text { Riesgo de } \\
\text { malnutrición }\end{array}$ & 84 & 65.63 \\
\hline Malnutrición & 5 & 3.91 \\
\hline \multicolumn{3}{|l|}{ Yesavage } \\
\hline $\begin{array}{l}\text { Sin } \\
\text { manifestaciones } \\
\text { depresivas }\end{array}$ & 59 & 46.09 \\
\hline $\begin{array}{l}\text { Con } \\
\text { manifestaciones } \\
\text { depresivas }\end{array}$ & 69 & 53.91 \\
\hline
\end{tabular}

Tabla 2. Asociación entre manifestaciones depresivas y estado nutricional

\begin{tabular}{|c|c|c|c|c|c|c|c|}
\hline \multirow[b]{3}{*}{$\begin{array}{l}\text { Manifestaciones } \\
\text { Depresivas }\end{array}$} & \multicolumn{7}{|c|}{ Estado Nutricional } \\
\hline & \multicolumn{2}{|c|}{ Normal } & \multicolumn{2}{|c|}{$\begin{array}{c}\text { Riesgo de } \\
\text { Malnutrición }\end{array}$} & \multicolumn{2}{|c|}{ Malnutrición } & \multirow[t]{2}{*}{$\mathbf{p}$} \\
\hline & $\mathbf{n}$ & $\%$ & $\mathbf{n}$ & $\%$ & $\mathbf{n}$ & $\%$ & \\
\hline $\begin{array}{l}\text { Sin } \\
\text { manifestaciones } \\
\text { depresivas }\end{array}$ & 30 & 50.85 & 26 & 44.07 & 3 & 5.08 & 0.001 \\
\hline $\begin{array}{l}\text { Con } \\
\text { manifestaciones } \\
\text { depresivas }\end{array}$ & 9 & 13.04 & 58 & 84.06 & 2 & 2.90 & \\
\hline
\end{tabular}

Por otro lado, se verificó la relación entre manifestaciones depresivas y estado nutricional ( $\mathrm{p}<0.001)$. El $84 \%$ de los adultos mayores con manifestaciones depresivas presentó riesgo de malnutrición (ver Tabla 2). 
Tabla 3: Fuerza de Asociación entre Manifestaciones depresivas y Estado Nutricional

\begin{tabular}{|c|c|c|c|c|c|}
\hline \multicolumn{2}{|c|}{$\begin{array}{l}\text { Estado nutricional } \\
\text { Manifestaciones }\end{array}$} & \multirow{2}{*}{$\begin{array}{c}\text { (OR) } \\
7.91\end{array}$} & \multirow{2}{*}{$\begin{array}{c}\mathbf{p}>\mathbf{I Z I} \\
0.000\end{array}$} & \multicolumn{2}{|c|}{ (IC:95\%) } \\
\hline \multicolumn{4}{|c|}{ depresivas } & & \\
\hline \multirow[t]{2}{*}{ Edad } & & 1.06 & 0.016 & 1.01 & 1.1 \\
\hline & No sabe & 16.79 & 0.03 & 1.29 & 217.1 \\
\hline Nivel & Primaria & 17.08 & 0.006 & 2.27 & 128.32 \\
\hline \multirow[t]{2}{*}{ Educati vo } & Secundaria & 9.97 & 0.023 & 1.36 & 72.64 \\
\hline & Superior & 19.56 & 0.005 & 2.44 & 156.57 \\
\hline
\end{tabular}

En la tabla 3, se muestran los resultados de la regresión logística ordinal a través del ajuste por variables confusoras encontrándose fuerza de asociación entre manifestaciones depresivas y estado nutricional $(\mathrm{p}<0.000)$, Indicando que los adultos mayores con manifestaciones depresivas tienen una probabilidad de 7.91 veces más de desarrollar malnutrición comparado con quien no presentó manifestaciones depresivas.

Como se puede observar en la tabla 3, el mayor riesgo de malnutrición está relacionado a un nivel educativo superior y primaria, para los individuos con nivel educativo (primaria y superior) el OR para malnutrición fue de 17.08 con una significancia de $(\mathrm{p}=0.006)$ y $19.56 \quad(\mathrm{p}=0.005)$ respectivamente.

\section{DISCUSIÓN}

El presente estudio encontró que en el grupo de adultos mayores institucionalizados, las personas con manifestaciones depresivas presentaron 7.91 veces más probabilidades de desarrollar malnutrición, a diferencia del grupo de adultos mayores que no las presentó. Este resultado promueve la necesidad de dar un mayor interés por parte del personal de salud y la población en general, a la presencia de manifestaciones depresivas con la finalidad de contribuir positivamente en una adecuada calidad de vida de la población adulto mayor institucionalizada.

Vinculado a lo anterior, en la revisión de evidencias científicas, no se encontraron estudios internacionales o nacionales que midieran la fuerza de asociación entre ambas variables, lo cual hace de este estudio una primera evidencia sobre la magnitud de la relación de interés. Sin embargo, existen estudios internacionales $(17,18)$ que asociaron ambas variables pero con un enfoque distinto, teniendo como factor causal al estado nutricional para desarrollar manifestaciones depresivas.

En México se indagó en la asociación entre ambas variables en población adulta mayor no institucionalizada reportándose fuerza de asociación estadísticamente significativa entre desnutrición y depresión a través de la prueba estadística de regresión logística ordinal (OR:2.4; $\mathrm{p}<0.001$ ), las personas adultas mayores no institucionalizadas con desnutrición presentan 2 veces más la probabilidad de manifestar depresión (17). De igual manera, en Madrid se evidenció que un elevado valor de MNA representa un factor de protección frente al riesgo de padecer depresión (OR:7; p 00.001). Es decir, los adultos mayores institucionalizados con desnutrición presentan 7 veces más la probabilidad de desarrollar depresión (18). Conectado y confirmando lo anterior, se reportó que el $38.2 \%$ de la población estudiada presentó simultáneamente síntomas depresivos y malnutrición. Los ancianos deprimidos presentaron un menor puntaje de MNA total $(16.3 \pm 3.2)$ que los no deprimidos $(21.9 \pm 3,3)(\mathrm{p}<0.001)(18)$.

Entre las debilidades del estudio se señala que, aunque realizado a través de un censo, los resultados obtenidos no se pueden generalizar debido a que la población estudiada es diferente a la realidad de condiciones de salud en comunidades. Sin embargo, estos hallazgos son epidemiológicamente válidos para poblaciones de adultos mayores institucionalizados. Igualmente, el diseño transversal no asegura temporalidad. Es decir, no se puede afirmar si la relación es causal; aunque, se consideró a la depresión como factor influyente sobre el estado nutricional en la población estudiada.

Igualmente, no se pudo determinar el tipo de malnutrición en los sujetos del estudio debido a que la herramienta para valorar el estado nutricional no incorpora datos bioquímicos, tales como la albúmina, hemoglobina, entre otros. No obstante, la herramienta utilizada es un tipo de 
tamizaje validado en población adulta mayor para identificar malnutrición.

\section{CONCLUSIONES}

Los resultados revelan la existencia de la relación entre ambas variables indicando, además, que la presencia de manifestaciones depresivas es un factor de riesgo frente al desarrollo del riesgo de malnutrición en la población adulta mayor institucionalizada. Esta evidencia permitiría que una intervención oportuna convierta el proceso de envejecimiento en un factor de desarrollo en los adultos mayores, reduciendo la prevalencia de desnutrición en la población adulta mayor y así aminorar los costos generados al gasto social del país.

Frente a estos resultados se deben implementar estrategias de salud para el adulto mayor mediante una atención integral, evaluando la presencia de manifestaciones depresivas como parte del tamizaje nutricional. Se recomienda la realización de estudios posteriores para prevenir y combatir la malnutrición de la población adulta mayor institucionalizada.

\section{AGRADECIMIENTOS}

Los autores agradecen a la Dra. Yessika Graya por su aporte metodológico desarrollado en los cursos de investigación de la UCSS y a Sor. Yrma Calderón por su aceptación y apoyo en la obtención de los datos del estudio.

\section{REFERENCIAS BIBLIOGRÁFICAS}

1. Organización Mundial de la Salud. Envejecimiento y Salud. Nota descriptiva $\mathrm{N}^{\mathrm{o}}$ 404. Septiembre 5. Varela L. Principios de Geriatría y 2015.

2. Organización Mundial de la Salud. Envejecimiento y salud [internet]. Ginebra, Suiza: OMS; 2017 [citado el 28 de febrero]. 6 Disponible en: http://www.who.int/mediacentre/fa ctsheets/fs 404/es/

3. Organización Mundial de la Salud. Envejecimiento y ciclo de vida [internet]. Ginebra, Suiza: OMS; 2017 [citado el 28 de febrero]. Disponible en: 7 http://www.who.int/ageing/about/f acts/es/

4. Organización Mundial de la Salud. Enfermedades crónicas y promoción de la salud [internet]. Ginebra, Suiza: OMS; 2017 [citado el 28 de febrero]. Disponible http://www.who.int/chp/chronic_d

isease_report/part 1/es/index8.htm Gerontología. 2da Edición. Lima, Universidad Peruana Cayetano Heredia; 2011.p.501- 512.

Cunha DF, Cunha SF, Unamuno MR, Vannucchi H. Serum le-vels assessment of Vitamina A, E, C, $\mathrm{B} 2$ and carotenoids in malnourished and nonmalnourished hospitalized elderly patients. Clinical Nutrition 2001; 20 (2): $\quad 167-70$.

Rodríguez D, del Castillo $\mathrm{P}$, Aguilar C, compiladores. Glosario de Términos en Salud Ambiental. 2da ed. Metepec: Centro Panamericano de Ecología 1990.

: 8uigoz Y, Lauque S, Vellas BJ. The Mini Nutritional Assessment. Perú: Centro Editorial de la 9 Humana y Salud. Programa de 12. Norma Técnica de Salud para la Salud Ambiental. OPS, OMS; Atención Integral de Salud de las
Identifying the elderly at risk of malnutrition. Clinical Geriatric Medicine 2002; 18 (4): 737-57.

World Health Organization. The World Health Report. Making a difference. Geneva: World Health Organization;

1999.

10. Hernandez Sampieri, Metodología de la Investigación 6ta edición . México,

2013.

11. Jorge Martínez de la Iglesia. Adaptación y validación al castellano del cuestionario de Pfeiffer (SPMSQ) para detectar la existencia de deterioro cognitivo en personas mayores de 65 años. Med Clin (Barc) 2001; 117: 129134).

Personas Adultas Mayores NTS $\mathrm{N}^{\circ} 043-2006 / \mathrm{MINSA}$ 
13. Jeanie De la Torre Maslucan y colaboradores. Validación de la versión reducida de la escala de depresión geriátrica en el consultorio externo de geriatría del Hospital Nacional Cayetano Heredia. Acta Med Per 23(3) 2006.

14. Guigoz Y, Vellas B, Garry PJ. Assessing the nutritional status of the elderly: The Mini Nutritional Assessment as part of the geriatric evaluation. Nutr Rev 1996; 54: S59-65.

15. Contreras y colaboradores. Malnutrición del adulto mayor y factores asociados en el distrito de Masma Chicche, Junín, Perú. RevMedHered. 2013; 24:186-191.

16. R.M 240-2013/MINSA. Guía técnica para la valoración nutricional antropométrica de la persona adulta mayor.

17. Pérez Cruz y colaboradores. Asociación entre desnutrición y depresión en el adulto mayor. NutrHosp. 2014； 29(4):901-906

18. Centurión y colaboradores. Asociación entre riesgo de desnutrición y capacidad afectiva en personas de edad avanzada institucionalizadas de la Comunidad de Madrid Nutr. Clín. diet. hosp. 2010; 30(2):33-4. 